\title{
Role of driving force on the clogging of inert particles in a bottleneck
}

\author{
Roberto Arévalo \\ CNR-SPIN, Dipartimento di Scienze Fisiche, Università di Napoli Federico II, I-80126, Napoli, Italy \\ Iker Zuriguel, ${ }^{*}$ Diego Maza, and Angel Garcimartín \\ Departamento de Física, Facultad de Ciencias, Universidad de Navarra, 31080 Pamplona, Spain
}

(Received 10 September 2013; published 18 April 2014)

\begin{abstract}
We present numerical results of the effect that the driving force has on the clogging probability of inert particles passing through a bottleneck. When the driving force is increased by four orders of magnitude, the mean avalanche size remains almost unaltered (increases 1.6 times) while the flow rate and the avalanche duration display strong dependence on this magnitude. This indicates that in order to characterize the ability of a system to $\operatorname{clog}$, the right variable to consider is the number of particles that pass through the outlet. The weak dependence of this magnitude on the driving force is explained in terms of the average kinetic energy of the flowing grains that has to be dissipated in order to get an arch stabilized.
\end{abstract}

DOI: 10.1103/PhysRevE.89.042205

PACS number(s): 45.70.-n, 89.40.-a

\section{INTRODUCTION}

When a group of particles pass through a bottleneck, clogging may happen if the size of the orifice is just a few times larger than the particles. Clogging, which is due to the development of arches that prevent the free movement of particles, is a generic phenomena with dramatic consequences in a wide variety of fields of science. The simplest example is a silo or the humble salt cellar, which has to be shaken to pour the salt. At a smaller spatial scale, clogging leads to intermittent flow when a dense suspension of microparticles passes through a constriction in a microchannel [1,2]. This behavior is also observed when electrons on the surface of liquid helium pass through nanoconstrictions [3,4]. Clogs of humans are also very important as they are behind tragedies like the one occurred at Hillsborough stadium (Sheffield, England) in 1989 [5].

Clogging in all these different systems shares strong similarities. For instance, it has been shown that a sufficiently high density of particles per unit area near the bottleneck is needed to observe clogging in suspensions [1,6], silos [7], and crowd dynamics [5]. Traditionally related with the density is the effect of pressure in clogging. Clogs of humans, indeed, are exclusively reported in panic situations, when people impulsively push each other in their wish to reach the exit. A practical solution which seems to release such pressure is the placement of an obstacle just in front of the outlet. Indeed, this strategy has been proved to be efficient in clogging prevention for both silos [8] and room evacuation [5,10-12].

Another feature that is found for the passage of particles through bottlenecks is the "faster-is-slower" behavior. For the case of humans, the social force model suggests that there is a nonmonotonous dependence of the flow rate on the desired velocity of the pedestrians $[5,9,13,14]$. The flow rate increases with the desired velocity for low values of this parameter but beyond some point, when the desired velocity is too high, the flow rate starts to decrease. Although this phenomenon is widely accepted, there is not solid experimental evidence of it [15]. For the case of ants, recent experiments show that the faster-is-slower effect does not exist [16].

\footnotetext{
*iker@unav.es
}

A behavior analogous to this has been found for suspensions of particles [1], but in this case the driving force is the pressure drop applied to suck the suspension. Interestingly, this behavior has never been reported for silos where the flow rate is proportional to the square root of the gravity $(\sqrt{g})$ [17]. This dependence was explained by assuming that the particles fall freely under the action of gravity from a height proportional to the size of the outlet [18]. Recent experimental results reported a dependence of the particle velocities on the outlet size, which is compatible with this idea [19], and a proof of the dependence of the flow rate on $\sqrt{g}$ by varying the force in a centrifuge [20]. Nevertheless, none of these experiments has studied systematically the effect of driving force on clogging. Given that clogging is a key ingredient to the eventual development of the "faster-is-slower" behavior, tackling this problem is of fundamental interest.

In this work, we report numerical simulations of the discharge of inert grains from a silo by gravity. We fixed the outlet size at 3.5 times the diameter of the particles to assure the development of clogs [21-27]. The flow rate and the avalanche size are measured for different gravities and the particles' mass. This allows us to prove that the avalanche size monotonously increases with the average kinetic energy of the particles at the exit, contradicting the faster-is-slower behavior.

\section{SIMULATIONS}

We use soft-particle molecular dynamics simulations of monosized disks in two dimensions. The restoring force in the normal direction of collision depends linearly on the particles overlap $\xi=d-r_{i j}$, with stiffness $k_{n}=10^{5}(\mathrm{mg} / \mathrm{d})$, where $d=1 \mathrm{~mm}$ is the particle diameter, $m$ is the mass, and $r_{i j}$ is the distance between the centers of the particles. Additionally, there is a dissipative force proportional to the relative normal velocity of the colliding grains, with damping parameter $\gamma_{n}=300(m \sqrt{g / d})$. We implement static friction placing a spring in the direction tangential to the normal joining of the centers of the particles [28]. The elongation of this spring is obtained by integrating the relative velocity of the surfaces in contact. The parameters are $k_{t}=(2 / 7) k_{n}$ and 
$\gamma_{t}=200(m \sqrt{g / d})$. The friction coefficient is set to $\mu=0.5$, and the gravity is set to $\Gamma$ times the values of $g$.

The simulation protocol is as follows. A flat-bottomed rectangular silo of width $18 d$ is filled with 35 layers of grains, which fall freely from a height much larger than their size. The side walls of the container are smooth, while the base is formed by fixed grains at mutual distances of $0.5 \mathrm{~d}$. Once the grains in the silo come to a rest, a hole is opened at the center of the base and the particles start to flow. The time at which each particle passes through the orifice is registered and these grains are relocated at the top of the silo in order to keep a constant height of the granular layer. A clog is detected when the kinetic energy of the system falls to the value it had before opening the hole (the difference amounts to several orders of magnitude) and remains under this value for 20000 time steps. Then the avalanche size, defined as the number of particles that flow through the outlet between two consecutive clogs, is measured. Finally, the flow is resumed by removing three of the grains conforming the blocking arch.

Several series of experiments (consisting of at least 1000 avalanches each) have been performed for different values of $\Gamma$. In order to optimize the computing time, we use a primary integration step $\delta=10^{-4} \sqrt{d / g}$ for simulations with $\Gamma \simeq 1$. For $\Gamma \simeq 3$ the integration step is reduced to $\delta / 3$, and for simulations at $\Gamma \simeq 6$ and $\Gamma \simeq 10$ we use a step of $\delta / 6$. For simulations at $\Gamma \simeq 10^{-3}$ we increase the time step to $10 \delta$.

\section{RESULTS}

In Fig. 1(a) we present the results of the average flow rates $\langle q\rangle$ obtained for particles with mass $m=1$ and different values of $\Gamma$. The flow rates are measured-in number of particles per second-for every avalanche discarding the first and last 10 particles of the avalanche. This is to avoid any possible influence of a transient regime, although we have checked that the results obtained considering the whole avalanche are essentially the same. Then, the average flow rate is calculated from all the avalanches obtained for the same value of $\Gamma$. As expected [17-20], the flow rate increases with $\sqrt{\Gamma}$. Remarkably, the deviations from this behavior are found to be negligible over four orders of magnitude of the driving force. From these results it can be concluded that the granular case does not show the "faster-is-slower" behavior.

The next step is exploring the effect of $\Gamma$ in clogging as "faster-is-slower" behavior is typically related with development of clogs and flow intermittencies. A readily accessible parameter that is related with clogging is the mean avalanche size $\langle s\rangle$. In fact, the distribution of avalanche (burst) sizes has been reported to decay exponentially both in the case of the silo [22] and in mice exiting a very small room [29]. This exponential-which can be explained by random alternation between particle and gap propagations [30] and also described in terms of a probabilistic model [22] - is characterized by the mean avalanche size $\langle s\rangle$. In Fig. 1(b) we report the results of the mean avalanche size $\langle s\rangle$ vs $\Gamma$. Interestingly, the dependence found is extremely weak: The driving force has to be increased in four orders of magnitude to obtain an augment of 1.6 times of $\langle s\rangle$. Indeed, the avalanche size is finite for $\Gamma \rightarrow 0$ (around 31 particles for this particular outlet size) [31]. Then, as $\Gamma$ is

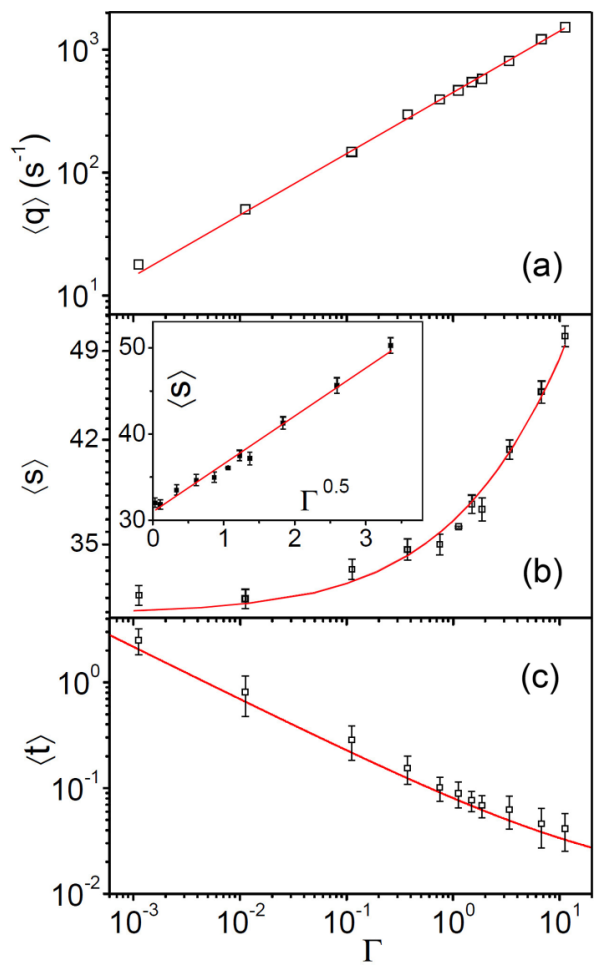

FIG. 1. (Color online) Logarithmic plots of (a) the average flow rate, (b) the mean avalanche size in number of particles, and (c) the mean avalanche duration vs the rescaled value of the acceleration $\Gamma$ for particles with mass $m=1$. In panel (a) the solid line represents a fit with the expression $\langle q\rangle=a \Gamma^{b}$ where $a=454 \pm 4$ and $b$ was set to $b=0.5$. In panel (b) the solid line represents a fit with the expression $\langle s\rangle=\left\langle s_{\Gamma \rightarrow 0}\right\rangle+c \Gamma^{d}$ where $\left\langle s_{\Gamma \rightarrow 0}\right\rangle=31.0 \pm 0.3, c=$ $5.5 \pm 0.4$, and $d=0.50 \pm 0.1$. In panel (c) the solid line is obtained from the fittings of panels (a) and (b) estimating $\langle t\rangle=\langle s\rangle /\langle q\rangle$ : $\langle t\rangle=0.0121+0.0683 \Gamma^{-0.5}$. In the inset of panel (b) the straight line obtained when representing $\langle s\rangle$ vs $\Gamma^{0.5}$ shows the goodness of this fit.

increased there is a monotonous augment of $\langle s\rangle$, which seems to be algebraic with an exponent of 0.5 .

If we consider the important dependence of the flow rate on $\Gamma$ and the (almost) negligible effect observed on the mean avalanche size, it follows that the mean avalanche duration $\langle t\rangle$ has to be modified accordingly. In Fig. 1(c) it is evidenced that the avalanche duration diverges towards infinite as $\Gamma \rightarrow 0$. Taking into account the fittings of Fig. 1(a) and Fig. 1(b), the functional dependence of $\langle t\rangle$ on $\Gamma$ can be estimated as $\langle t\rangle=\langle s\rangle /\langle q\rangle$ which is depicted in Fig. 1(c).

From the results displayed in Fig. 1, it can be concluded that clogging is controlled, primarily, by geometrical factors. Surely, despite the evident influence that changing the gravity has on the flow rate and the contact forces [32], the avalanche size distribution is barely affected. This suggests that the number of beads passing through the orifice is the relevant magnitude in order to characterize the clogging configurations occurring above the outlet. On the contrary, the duration of the avalanche by itself does not seem to be a useful magnitude to describe the clogging process as it is strongly affected by the flow rate. Having said that, it is obvious that the avalanche size 

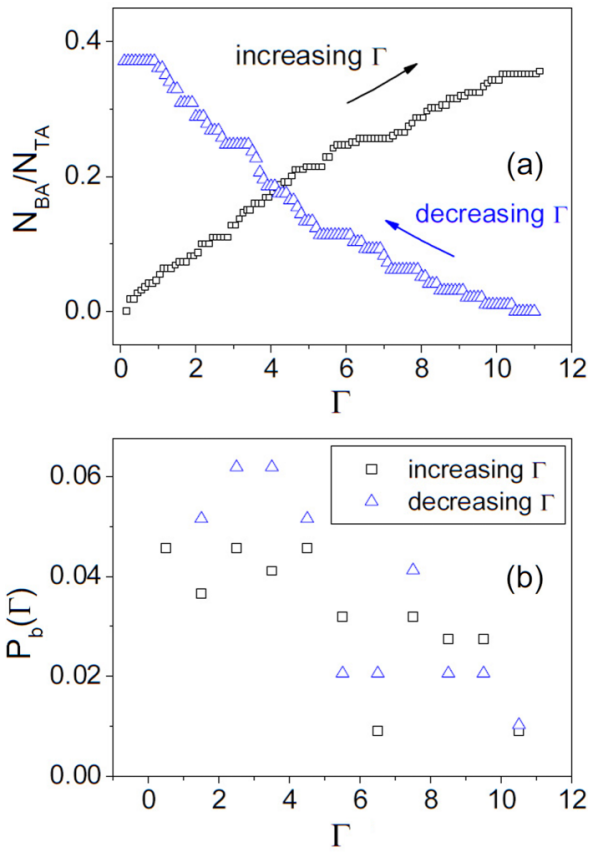

FIG. 2. (Color online) (a) Number of broken arches $\left(N_{B} A\right)$ with respect to the initial number of arches analyzed $\left(N_{B} A\right)$. Squares $(\square)$ represent the results obtained when $\Gamma$ is increased from 0.11 to 11 and triangles $(\triangle)$ represent the results obtained when $\Gamma$ is reduced from 11 to 0.11 . In all the cases the mass of the particles was $m=1$. (b) Probability that an arch is broken at a given value of $\Gamma$ for ( $\square$ ) increasing and $(\triangle)$ decreasing $\Gamma$.

is also weakly affected by the gravity, and in what follows we investigate the physical mechanisms behind this behavior.

A first hypothesis that we consider is that arches could form independently on $\Gamma$ but only some of them are able to resist the weight of the granular layer when gravity is increased. With the aim of checking whether this idea is plausible, we carried out additional simulations to check the stability of the arches formed at $\Gamma=0.1$. The protocol is the same as the one explained above, but once an arch is formed, we do not destroy it. Instead, we quasistatically increase gravity in steps $\Delta g=$ 0.001 each 20000 time steps. If the arch breaks spontaneously, we record the value of $\Gamma$, and a new experience is performed setting back gravity at $\Gamma=0.1$. In order to avoid prohibitively long runs, if the arches do not break spontaneously, the runs are stopped for $\Gamma=11$. Another series of experiments is performed using the same protocol but decreasing $\Gamma$; i.e., we take arches initially formed at $\Gamma=11$ and reduce this value up to $\Gamma=0.1$ (minimum). In both series of simulations the stability of around 300 arches is analyzed. In Fig. 2(a) we display the number of broken arches $\left(N_{B A}\right)$ divided by the total number of initial arches analyzed $\left(N_{T A}\right)$ when increasing and decreasing $\Gamma$. From these results, the probability that an arch is broken at a given $\Gamma$ can be easily obtained [Fig. 2(b)]. Surprisingly, the outcomes obtained for both processes are very similar. The probability of breaking an arch decreases when the gravity is increased. This implies that the increase of the mean avalanche size with $\Gamma$ obtained in Fig. 1(b) cannot be attributed to an arch stability that depends on $\Gamma$. On the contrary, as the gravity is greater, the arches become stronger.

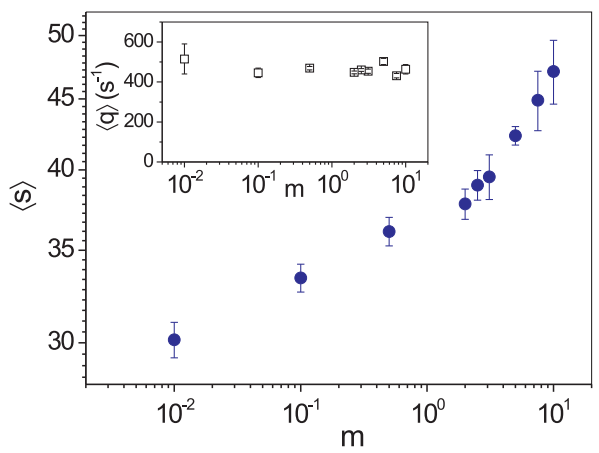

FIG. 3. (Color online) Logarithmic plot of the mean avalanche size vs the mass of the particles obtained for $\Gamma=1$. Inset: lin-log plot of the flow rate, in number of particles per second, for $\Gamma=1$ and different values of the mass of the particles.

Another hypothesis to explain the increase of the avalanche size with gravity displayed in Fig. 1(b) can be based in kinematic effects. Increasing the gravity leads to an augment of the velocity of the particles $(v)$ in the same proportion than the flow rate $(q \propto v \propto \sqrt{g})$ as evidenced in the inset of Fig. 4 and previously reported in Refs. [17,19,20]. Thus, the velocity increase could lead to a reduction of the probability of forming an arch, with the corresponding increase of the avalanche size. Indeed, the velocity of the particles determines the time that the particles spend in the region of clogging. But it also affects the momentum of the particles and their kinetic energy near the orifice. In order to unveil which of these parameters (velocity, momentum, or kinetic energy) is behind the clogging reduction, we performed simulations varying the mass of the particles and keeping constant the stiffness [33] and $\Gamma=1$. As expected, the flow rate in number of particles per second does not depend significatively on $m$ (inset, Fig. 3). Nevertheless, the avalanche size increases monotonously with $m$, revealing that clogging depends on this parameter in a way similar to the way it does on the gravity.

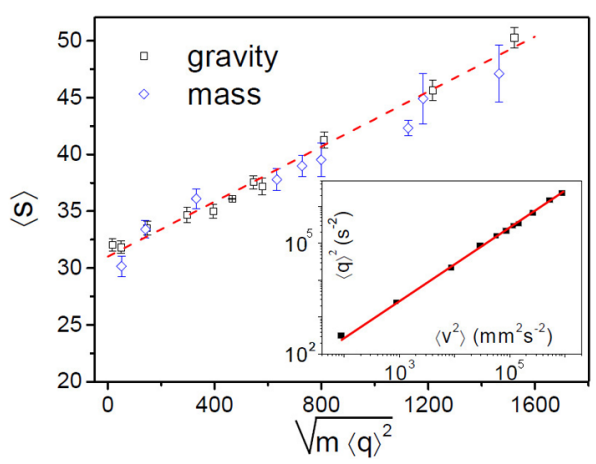

FIG. 4. (Color online) Mean avalanche size, measured in number of particles, vs $\sqrt{m\langle q\rangle^{2}}$. As shown in the inset $\langle q\rangle^{2} \propto\left\langle v^{2}\right\rangle$, where $v$ is the velocity of the particles at the orifice. Then, $m\langle q\rangle^{2}$ is proportional to the kinetic energy of the particles at the orifice. Squares $(\square)$ represent the results obtained when varying $\Gamma$ (and then $q$ ) and diamonds $(\diamond)$ represent those when varying the mass of the particles. The dashed line corresponds to the following expression: $\langle s\rangle=31+0.0121 \sqrt{m\langle q\rangle^{2}}$ which is obtained from the fitting expressions of Figs. 1(a) and 1(b). 
In Fig. 4 we present all the results of $\langle s\rangle$ vs $\sqrt{m\langle q\rangle^{2}}$, which is proportional to the square root of the kinetic energy of the particles at the exit as $\langle q\rangle^{2} \propto\left\langle v^{2}\right\rangle$ (inset of Fig. 4). The good collapse obtained-which is not achieved when plotting $\langle s\rangle$ vs $m\langle q\rangle$-strongly suggests that the physical magnitude behind clogging reduction by increasing gravity or particle density is the averaged kinetic energy per particle. This dependence can be explained because in order to get an arch completely stabilized, the kinetic energy of all the particles in the system has to be dissipated through collisions. Assuming that (1) the kinetic energy of the grains is related to the total kinetic energy in the system, (2) the higher the kinetic energy, the longer it takes to the system to freeze, and (3) during this time is when an arch can be destabilized, it seems reasonable that increasing the kinetic energy augments the avalanche size.

\section{CONCLUSIONS}

In summary, we report numerical results of the flow rate and clogging of inert particles through an orifice when varying the driving force. We show that as the gravity increases, the flow rate becomes faster and the avalanche duration becomes smaller. The avalanche size, measured in number of grains, shows a very weak increase with gravity, which is attributed to the time that the system needs to dissipate all its kinetic energy. These results seem to contradict the "faster-is-slower" behavior, at least for the case of inert grains discharged from a silo. At this point it should be stressed that there is a crucial difference among the system explored in this work and the flow through bottlenecks of suspensions or pedestrians. Once a clog is formed, and all the energy in the system is dissipated, clogs in a silo are forever stable. Complete dissipation of energy, however, cannot be attained in suspensions (where clogs can be broken by the perturbations introduced by the fluid) or active matter (where clogs break up without external energy supply). Having this in mind, the results of this work suggest that "faster-is-slower" behavior (if it exists) should be related to the ability of the system to break up the clogs. Indeed, if the probability that an arch is broken decreases with $\Gamma$ (as shown in Fig. 2), in clogs of suspensions or active matter it is expected that an increase of the driving force would lead to an augment of the time needed to resume the flow once a clog is formed. This idea has been already suggested for the case of suspensions [34] and vibrated silos [35]. The latter resemble suspensions and active matter as complete dissipation of energy cannot be attained and arches can be shattered. The fact that in vibrated silos with small orifices the flow rate is mainly governed by the unclogging time $[36,37]$, gives robustness to this proposal.

\section{ACKNOWLEDGMENTS}

We thank Raúl Cruz Hidalgo and Luis Ariel Pugnaloni for their useful comments. This work has been financially supported by Projects FIS2011-26675 (Spanish government) and PIUNA (Universidad de Navarra). R.A. thanks MIURFIRB RBFR081IUK for financial support.
[1] M. D. Haw, Phys. Rev. Lett. 92, 185506 (2004).

[2] D. Genovese and J. Sprakel, Soft Matter 7, 3889 (2011).

[3] D. G. Rees, I. Kuroda, C. A. Marrache-Kikuchi, M. Höfer, P. Leiderer, and K. Kono, Phys. Rev. Lett. 106, 026803 (2011).

[4] D. G. Rees, H. Totsuji, and K. Kono, Phys. Rev. Lett. 108, 176801 (2012).

[5] D. Helbing et al., Transport. Sci. 39, 1 (2005).

[6] A. Guariguata, M. A. Pascall, M. W. Gilmer, A. K. Sum, E. D. Sloan, C. A. Koh, and D. T. Wu, Phys. Rev. E 86, 061311 (2012).

[7] N. Roussel, T. L. H. Nguyen, and P. Coussot, Phys. Rev. Lett. 98, 114502 (2007).

[8] I. Zuriguel, A. Janda, A. Garcimartín, C. Lozano, R. Arévalo, and D. Maza, Phys. Rev. Lett. 107, 278001 (2011).

[9] D. Helbing, I. Farkas, and T. Vicsek, Nature (London) 407, 487 (2000).

[10] D. Yanagisawa, A. Kimura, A. Tomoeda, R. Nishi, Y. Suma, K. Ohtsuka, and K. Nishinari, Phys. Rev. E 80, 036110 (2009).

[11] N. Shiwakoti, M. Sarvi, G. Rose, and M. Burd, Transport. Res. Rec. 2196, 176 (2010).

[12] A. Kirchner, K. Nishinari, and A. Schadschneider, Phys. Rev. E 67, 056122 (2003).

[13] D. R. Parisi and C. O. Dorso, Physica A (Amsterdam, Neth.) 354, 606 (2005).

[14] K. Suzuno, A. Tomoeda, and D. Ueyama, Phys. Rev. E 88, 052813 (2013).

[15] A. Seyfried, O. Passon, B. Steffen, M. Boltes, T. Rupprecht, and W. Klingsch, Transport. Sci. 43, 395 (2009).
[16] S. Boari, R. Josens, and D. R. Parisi, PLoS ONE 8, e81082 (2013).

[17] W. A. Beverloo, H. A. Leniger, and J. J. Van de Velde, Chem. Eng. Sci. 15, 260 (1961).

[18] R. L. Brown and J. C. Richards, Principles of Powder Mechanics (Pergamon Press, Oxford, 1970).

[19] A. Janda, I. Zuriguel, and D. Maza, Phys. Rev. Lett. 108, 248001 (2012).

[20] S. Dorbolo et al., Gran. Matt. 15, 263 (2013).

[21] K. To, P. Y. Lai, and H. K. Pak, Phys. Rev. Lett. 86, 71 (2001); Phys. Rev. E 71, 060301(R) (2005).

[22] I. Zuriguel, A. Garcimartín, D. Maza, L. A. Pugnaloni, and J. M. Pastor, Phys. Rev. E 71, 051303 (2005); I. Zuriguel, L. A. Pugnaloni, A. Garcimartín, and D. Maza, ibid. 68, 030301(R) (2003).

[23] A. Janda et al., Europhys. Lett. 84, 44002 (2008).

[24] J. Tang and R. P. Behringer, Chaos 21, 041107 (2011).

[25] L. Kondic, Gran. Matt. 16, 235 (2014).

[26] C. C. Thomas and D. J. Durian, Phys. Rev. E 87, 052201 (2013).

[27] S. Tewari, M. Dichter, and B. Chakraborty, Soft Matter 9, 5016 (2013).

[28] R. Arévalo, D. Maza, and L. A. Pugnaloni, Phys. Rev. E 74, 021303 (2006).

[29] C. Saloma et al., Proc. Nat. Acad. Sci. USA 100, 11947 (2003).

[30] D. Helbing, A. Johansson, J. Mathiesen, M. H. Jensen, and A. Hansen, Phys. Rev. Lett. 97, 168001 (2006).

[31] Note that the extrapolation to $\Gamma=0$ makes no sense as the flow rate would be zero and the avalanche would not be defined. 
[32] See Supplemental Material at http://link.aps.org/supplemental/ 10.1103/PhysRevE.89.042205 for a movie of the discharge of two identical silos with $\Gamma=11$ and $\Gamma=0.1$.

[33] In this set of simulations we take into account the fact that when the mass is set to a new value $m^{*}=\alpha m$ while keeping the same stiffness, the contact duration is changed to $t_{c}^{*}=\sqrt{\alpha} t_{c}$. This in turn, requires $\gamma_{n}^{*}=\sqrt{\alpha} \gamma_{n}$ to keep the restitution coefficient unchanged.
[34] T. W. Muecke, J. Petrol. Technol. 31, 144 (1979).

[35] J. R. Valdes and J. C. Santamarina, Can. Geotech. J. 45, 177 (2008).

[36] A. Janda et al., Europhys. Lett. 87, 24002 (2009).

[37] P. A. Gago, D. R. Parisi, and L. A. Pugnaloni, Traffic and Granular Flow '11, edited by V. V. Kozlov, A. P. Buslaev, A. S. Bugaev, M. V. Yashina, A. Schadschneider, and M. Schreckenberg (Springer, Berlin, Heidelberg, 2013). 